\title{
Administration of Micronized Purified Flavonoid Fraction During Sclerotherapy of Reticular Veins and Telangiectasias: Results of the National, Multicenter, Observational Program VEIN ACT PROLONGED-C1
}

Vadim Y. Bogachev (D) B Boris V. Boldin · Pavel Y. Turkin

Received: April 11, 2018 / Published online: June 15, 2018

(C) The Author(s) 2018

\section{ABSTRACT}

Introduction: Sclerotherapy is a common technique for the removal of intradermal veins. This study examined the rationale for prescribing micronized purified flavonoid fraction (MPFF) in clinical, etiological, anatomic, pathophysiologic (CEAP) class C1 patients with dilated intradermal veins scheduled for sclerotherapy.

Methods: In a national, multicenter, observational program, physicians recruited CEAP C1s patients scheduled for sclerotherapy. The decision to prescribe adjuvant MPFF (1000 mg/day for 6 weeks beginning 2 weeks before sclerotherapy) was made according to usual practice. Disease severity and treatment outcomes were assessed at baseline and 4 weeks post-sclerotherapy using a visual analog scale (VAS) as well as quality-of-life (CIVIQ-14) and patient satisfaction (Darvall) questionnaires.

Results: A total of 70 physicians recruited 1150 patients: 1071 (93\%) women, 79 (7\%) men. Mean age $( \pm \mathrm{SD})$ was $40.7 \pm 10.7$ years (range

Enhanced digital features To view enhanced digital features for this article go to https://doi.org/10.6084/ m9.figshare.6396236.

V. Y. Bogachev $(\bowtie) \cdot$ B. V. Boldin · P. Y. Turkin Department of Faculty Surgery No. 2, Pirogov Russian National Research Medical University, Moscow, Russia

e-mail: Vadim.bogachev63@gmail.com
18-74) and mean body mass index was $23.6 \pm 3.3 \mathrm{~kg} / \mathrm{m}^{2}$. Reticular veins were observed in $42.1 \%$ of patients and $57.9 \%$ had telangiectasias. MPPF was prescribed to 905 patients (79\%). Sclerotherapy was associated with statistically significant decreases in mean VAS scores for leg heaviness, pain, sensation of swelling, night cramps, and itching. For each symptom, MPFF-treated patients showed a more pronounced improvement than those undergoing sclerotherapy alone: mean VAS pain score with MPFF decreased from $1.90 \pm 2.30$ to $0.30 \pm 0.62$ versus $1.72 \pm 1.93$ to $0.52 \pm 0.99$ with sclerotherapy alone; mean VAS leg heaviness score with MPFF decreased from $2.80 \pm 2.43$ to $0.47 \pm 1.07$ versus $2.38 \pm 2.23$ to $0.76 \pm 0.85$ with sclerotherapy alone. Patient quality-of-life indicators improved with symptom resolution, particularly pain, and for each indicator the observed improvement was greater with MPFF. The outcomes of treatment exceeded patient expectations. Fewer patients experienced sclerotherapy-induced hyperpigmentation with adjunctive MPFF versus sclerotherapy alone $(33.9 \%$ versus $41.2 \%$, respectively, $P=0.034$ ). No adverse events related to MPFF were observed.

Conclusion: Resolution of venous symptoms post-sclerotherapy was greater in patients treated with MPFF compared with those undergoing sclerotherapy alone, supporting the rationale for use of MPFF in patients undergoing sclerotherapy. Funding: Servier. 
Keywords: Micronized purified flavonoid fraction; MPFF; Reticular veins; Sclerotherapy; Telangiectasias

\section{INTRODUCTION}

Dilated intradermal reticular veins and telangiectasias in their isolated form [clinical, etiological, anatomic, pathophysiologic (CEAP) class C1] are found in $25 \%$ of working-age people, and in almost $70 \%$ of individuals with more severe grades of chronic vein disease (CVD). Reticular veins and telangiectasias occur predominantly in women and have long been regarded by the medical community as a cosmetic defect. However, numerous epidemiological studies have shown a clear relationship between dilated intradermal veins and diseasespecific complaints, the frequency of which varies from $15 \%$ to $50 \%[1,2]$. They are also associated with a significantly reduced quality of life (QoL), and can be a source of intradermal hemorrhages and external bleeding $[1,2]$.

The main technique for the removal of intradermal veins is compression sclerotherapy. This involves intravenous injection of solutions that cause chemical burning and destruction of the endothelium, which leads to obliteration of the target vessel. However, phlebosclerosing treatment of reticular veins and telangiectasias can be associated with a variety of undesirable side reactions, including the formation of ecchymoses and the development of hyperpigmentation and neovasculogenesis (so-called matting). One method that has been proposed to reduce these effects is based on the use of drugs that modulate the consequences of damage to the endothelium, and local inflammatory reactions [3].

Micronized purified flavonoid fraction (MPFF) is an established venous active drug, and its marketed formulation (Detralex ${ }^{\circledR}$ and other trade names) contains 90\% diosmin (450 mg) and $10 \%$ other flavonoids (50 mg) expressed as hesperidin given orally twice daily. It has a proven record of effectiveness in improving leg symptoms in patients with CVD and venous edema. Over the past 5-6 years, several pub- lished studies have demonstrated that treatment with MPFF is able to reduce the incidence of complications associated with endovascular phlebosclerosing treatment. Unfortunately, the number of patients participating in these studies was small, and their designs were varied [4-6]. The current, national, multicenter program was conducted to evaluate the efficacy and feasibility of MPFF treatment in patients undergoing phlebosclerosing procedures for reticular veins and telangiectasias in routine clinical practice.

\section{METHODS}

Between March and September 2016, participating physicians (all specialists in phlebology) from 31 regions and 60 cities in the Russian Federation recruited patients with a CEAP C1s classification who were scheduled for phlebosclerosing treatment. The protocol and design of the study were approved by the local ethics committee of the Russian National Research Medical University named after N.I. Pirogov (Chairmen Storozhakov G.I.) (extract from Protocol No. 144 dd. 26.01.2016). In accordance with the Federal Law No. 61-FZ, the study did not require the approval of the Ethics Council of the Ministry of Health of the Russian Federation and permission from the Ministry of Health of Russia, as this study had an observational nature. The study was conducted in accordance with the legislative requirements and ethical principles set forth in the Federal Law "On the circulation of medicines" (No. 61-FZ of April 12, 2010, as amended), National Standard of the Russian Federation GOST R 52379-2005 “Good Clinical Practice", World Medical Association's Declaration of Helsinki (1964, with subsequent amendments), and the guidelines for Good Clinical Practice (ICH GCP).

All subjects were required to be over 18 years of age, with a CEAP C1s classification, no known allergy to anesthetics, MPFF or sclerosing agents, and no history of phlebotropic agent use in the 4 weeks before selection. Subjects were excluded if they had concomitant disease(s) that might interfere with the results 
(diabetes, hypertension, heart failure, arterial disease, deep or superficial thrombophlebitis, renal insufficiency, malignant disease, connective tissue disorders), any infection within the last 6 weeks, and a BMI greater than $30 \mathrm{~kg} / \mathrm{m}^{2}$. Pregnant or breastfeeding women, or women wishing to become pregnant during the course of the study were also excluded.

Three visits to the study center were scheduled: (1) inclusion visit; (2) on the day of sclerotherapy (approximately 2 weeks after the inclusion visit); and (3) follow-up visit (34 weeks after the end of the phlebosclerosing treatment). The decision to prescribe MPFF at a daily dose of $1000 \mathrm{mg}$ was taken by the physicians themselves on the basis of the individual patient. When prescribed, MPFF was initiated 2 weeks before the sclerotherapy and continued for 6 weeks.

A number of instruments were used to assess disease severity and treatment outcomes. Symptom severity and changes in vein-specific symptoms were estimated with a $10-\mathrm{cm}$ visual analog scale (VAS). QoL indicators and patient satisfaction with treatment outcomes were assessed with the disease-specific questionnaires CIVIQ-14 (ChronIc Venous Insufficiency quality of life Questionnaire, 14 items) $[7,8]$ and Darvall [9]. CIVIQ-14 consists of 14 items in three domains: pain (three items), physical (five items), and psychological (six items) each with five possible answers (1-5). The scores for CIVIQ-14 can be calculated for each of the three dimensions and as a global index score. Change in CVD clinical class was determined using the CEAP classification [10].

Statistical analyses were conducted using the SPSS 16.0 software. When comparing parameters before and after sclerotherapy, the Student's $t$ test for paired samples was used.

When comparing groups of patients with or without MPFF, the Student's $t$ test for independent samples was used. For all statistical criteria, the level of significance was set at 0.05 .

\section{RESULTS}

A total of 70 physicians recruited 1150 patients with a CEAP C1s classification who were scheduled for sclerotherapy. The cohort comprised 79 (7\%) men and 1071 (93\%) women. Mean age $( \pm$ SD) was $40.7 \pm 10.7$ years (range 18-74 years). Most patients were in the 30-39year (32.6\%) and 40-49-year age groups (29.3\%), and $95.6 \%$ were less than 60 years old. Mean (SD) body mass index (BMI) was $23.6 \pm 3.3 \mathrm{~kg} / \mathrm{m}^{2}$. Around two-thirds [753 $(65.5 \%)]$ of patients had a normal BMI, 319 (27.7\%) were overweight, 39 (3.4\%) were obese, and $33(2.9 \%)$ were underweight. Additional CVD risk factors identified in the study are shown in Table 1. Reticular veins were observed in $42.1 \%$ of respondents and telangiectasias were present in $57.9 \%$ of cases. In the MPFF group, 129 patients (14.3\%) had no vein-specific complaints (leg heaviness, pain, sensation of swelling, night cramps, and itching) and in the control group there were 28 such patients (11.4\%); this difference was not statistically significant. All patients underwent sclerotherapy; the sclerosant used was Fibro-Vein in 585 patients $(50.9 \%)$ and polidocanol (Ethoxysclerol) in $565(49.1 \%)$ patients. MPFF was

Table 1 CVD risk factors present among patients in the study $(n=1150)$

\begin{tabular}{ll}
\hline Risk factor & Number (\%) \\
\hline Female gender & $1071(93.1)$ \\
Family history of CVD & $862(74.9)$ \\
Excessive body mass or obesity & $358(31.1)$ \\
Use of hormonal contraception or HRT & $198(17.2)$ \\
Childbirth & $931(86.9)$ \\
2 or more & $521(48.6)$ \\
Current smoker & $163(14.2)$ \\
No regular exercise & $762(66.3)$ \\
History of thrombosis or thrombophlebitis & $7(0.6)$ \\
Mean (SD) hours/day spent & \\
Standing & $5.6 \pm 3.0$ \\
Sitting & $5.5 \pm 2.5$ \\
\hline
\end{tabular}

$C V D$ chronic venous disease, $H R T$ hormone replacement treatment 
Table 2 Severity of vein-specific complaints on the 10-cm visual analog scale before and 1 month after sclerotherapy

\begin{tabular}{|c|c|c|c|c|c|}
\hline \multirow{3}{*}{$\begin{array}{l}\text { Severity of } \\
\text { complaints } \\
\text { according to VAS }\end{array}$} & \multicolumn{4}{|l|}{ Mean score \pm SD } & \multirow{3}{*}{$\begin{array}{l}P \text { value } \\
\text { (MPFF vs } \\
\text { control } \\
\text { after therapy) }\end{array}$} \\
\hline & \multicolumn{2}{|l|}{ Before } & \multicolumn{2}{|l|}{ After } & \\
\hline & MPFF $(n=905)$ & Control $(n=245)$ & MPFF $(n=905)$ & Control $(n=245)$ & \\
\hline Leg heaviness & $2.80 \pm 2.43$ & $2.38 \pm 2.23$ & $0.47 \pm 1.07$ & $0.76 \pm 0.85$ & $<0.001$ \\
\hline Pain & $1.90 \pm 2.30$ & $1.72 \pm 1.93$ & $0.30 \pm 0.62$ & $0.52 \pm 0.99$ & $<0.001$ \\
\hline Sensation of swelling & $1.64 \pm 2.20$ & $1.50 \pm 1.94$ & $0.24 \pm 0.62$ & $0.38 \pm 0.82$ & $<0.005$ \\
\hline Night cramps & $0.84 \pm 1.67$ & $0.47 \pm 1.22$ & $0.05 \pm 0.35$ & $0.16 \pm 0.68$ & $<0.001$ \\
\hline Itching & $0.55 \pm 1.60$ & $0.15 \pm 0.55$ & $0.05 \pm 0.43$ & $0.15 \pm 0.72$ & 0.007 \\
\hline
\end{tabular}

In both groups all parameters showed a statistically significant $(P<0.001)$ decrease (improvement) after therapy with MPFF

prescribed as adjuvant treatment for 60 days to 905 patients $(79 \%)$.

At the follow-up visit approximately 1 month after sclerotherapy, a statistically significant decrease in mean scores for all veinspecific complaints was recorded including leg heaviness, pain, sensation of swelling, night cramps, and itching (Table 2, Fig. 1). The reduction in intensity of symptoms post-sclerotherapy was observed in both the MPFF and sclerotherapy alone (control) groups, but for each complaint a more pronounced improvement was observed in patients who underwent sclerotherapy in conjunction with MPFF

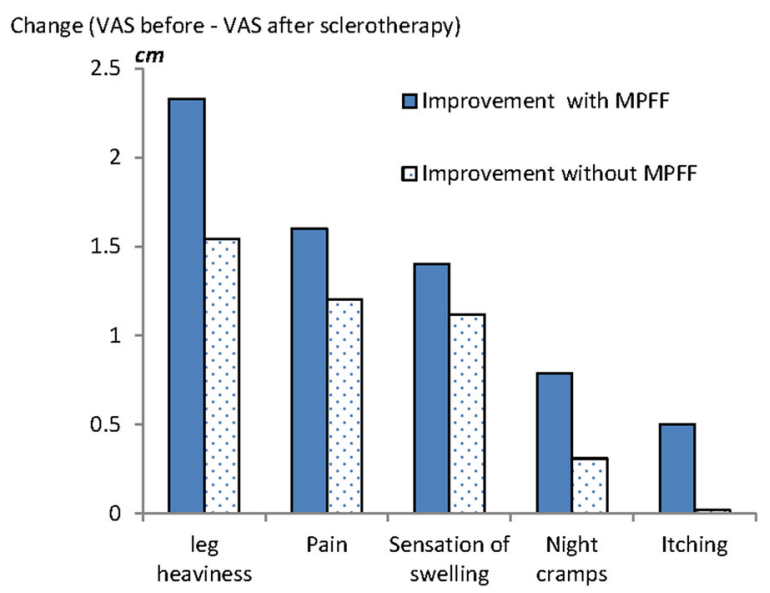

Fig. 1 Improvement in severity of vein-specific complaints assessed on a $10-\mathrm{cm}$ visual analog scale (VAS) in patients treated/untreated with MPFF
(Table 2, Fig. 1). For pain, the mean VAS score decreased from $1.90 \pm 2.30$ to $0.30 \pm 0.62$ in patients who received MPFF and from $1.72 \pm 1.93$ to $0.52 \pm 0.99$ in patients with sclerotherapy alone. Similarly, leg heaviness decreased from $2.80 \pm 2.43$ to $0.47 \pm 1.07$ in patients who received MPFF and from $2.38 \pm 2.23$ to $0.76 \pm 0.85$ in patients treated with sclerotherapy alone. Greater improvements in symptom severity with MPFF were also observed for sensation of swelling, night cramps, and itching (Table 2).

The reduction in severity of vein-specific complaints was strongly correlated with an improvement in CIVIQ-14 QoL indicators in both the MPFF and control groups, but for each indicator the improvement was greater among patients who received MPFF (Table 3). This was particularly noticeable for the CIVIQ-14 pain score, which improved from a mean of 18.8 preprocedure to 4.2 post-procedure in patients who underwent sclerotherapy in conjunction with MPFF compared with an improvement from 18.2 to 7.4 in patients undergoing sclerotherapy alone (Table 3). The CIVIQ-14 global index score at the final examination improved more than fourfold with MPFF from $13.4 \pm 12.5$ to $3.0 \pm 4.6$. With sclerotherapy alone the global index score improved from $12.2 \pm 12.0$ to $5.3 \pm 6.8$. A reduction in the global index score indicates an improved QoL. According to the Darvall questionnaire, the outcomes following 
Table 3 Mean scores for CIVIQ-14 quality-of-life indicators before and 1 month after sclerotherapy

\begin{tabular}{|c|c|c|c|c|c|}
\hline \multirow[t]{3}{*}{ CIVIQ-14 scores } & \multicolumn{4}{|l|}{ Mean score \pm SD } & \multirow{3}{*}{$\begin{array}{l}P \text { value } \\
\text { (MPFF vs control } \\
\text { after therapy) }\end{array}$} \\
\hline & \multicolumn{2}{|l|}{ Before } & \multicolumn{2}{|l|}{ After } & \\
\hline & MPFF $(n=905)$ & Control $(n=245)$ & MPFF $(n=905)$ & Control $(n=245)$ & \\
\hline Pain & $18.8 \pm 17.4$ & $18.2 \pm 16.0$ & $4.2 \pm 6.5$ & $7.4 \pm 9.8$ & $<0.001$ \\
\hline Physical & $8.0 \pm 12.2$ & $8.1 \pm 12.4$ & $2.3 \pm 5.1$ & $3.7 \pm 8.1$ & $<0.001$ \\
\hline Psychological & $12.5 \pm 14.1$ & $10.4 \pm 12.2$ & $2.6 \pm 5.2$ & $4.7 \pm 7.6$ & $<0.001$ \\
\hline Global & $13.4 \pm 12.5$ & $12.2 \pm 12.0$ & $3.0 \pm 4.6$ & $5.3 \pm 6.8$ & $<0.001$ \\
\hline
\end{tabular}

In the disease-specific questionnaire CIVIQ-14, the lower the score, the better the quality of life

the intervention significantly exceeded patients' expectations in all dimensions (appearance, clothes/ability to wear short skirts, improvement in communication, social and leisure activities).

At the end of the follow-up period the number of respondents with CEAP class COa (i.e., no visible signs or symptoms) had increased fourfold.

The incidence of adverse reactions typically associated with phlebosclerosing treatment is presented in Table 4. Fewer patients experienced sclerotherapy-induced hyperpigmentation with adjunctive MPFF versus sclerotherapy alone $(33.9 \%$ versus $41.2 \%$, respectively, $P=0.034)$. No adverse events related to MPFF treatment were observed.

Table 4 Comparative assessment of adverse reactions associated with phlebosclerosing treatment in patients who were treated or not treated with MPFF

\begin{tabular}{|c|c|c|c|c|c|}
\hline & \multicolumn{2}{|c|}{$\begin{array}{l}\text { Patients } \\
\text { treated with } \\
\text { MPFF for } \\
60 \text { days } \\
(n=905)\end{array}$} & \multicolumn{2}{|c|}{$\begin{array}{l}\text { Patients not } \\
\text { treated with } \\
\text { MPFF } \\
(n=245)\end{array}$} & \multirow[t]{2}{*}{$P$ value } \\
\hline & $n$ & $\%$ & $n$ & $\%$ & \\
\hline Phlebitis & 68 & 7.5 & 22 & 9.0 & 0.449 \\
\hline Pigmentation & 307 & 33.9 & 101 & 41.2 & $0.034^{*}$ \\
\hline Neoangiogenesis & 40 & 4.4 & 16 & 6.5 & 0.173 \\
\hline Necrosis & 6 & 0.7 & 0 & 0.0 & 0.201 \\
\hline
\end{tabular}

${ }^{*}$ Differences statistically significant

\section{DISCUSSION}

Recruitment of patients in this study was limited to those with a CEAP C1 classification of CVD. However, the risk factors for dilation of the intradermal veins identified in the current study were identical to those associated with the development of more severe forms of CVD. As such, reticular veins and telangiectasias should be considered not as a separate clinical entity but as a variant of the progressive course of CVD with a high probability of an increase in clinical class in the future. This statement may be justified indirectly by the complaints of patients in the current study, which coincide with the vein-specific symptoms typical of more severe forms of CVD. The disappearance of veinspecific symptoms after sclero-obliteration of pathologically altered intradermal veins is therefore predictable, and confirms that compression sclerotherapy is not only a cosmetic procedure but also a fully fledged treatment method.

There is considerable rationale for the use of adjuvant phlebotrophic therapy during phlebosclerosing treatment. It targets adverse effects associated with vein-specific inflammation by inhibiting leukocyte-endothelial interactions and a number of studies have shown that MPFF can improve outcomes following surgical and endovascular interventions for CVD of the lower limbs [11, 12]. In the current study, patients in the sclerotherapy with adjunctive MPFF group had a greater reduction in all venous complaints including leg heaviness, 
pain, sensation of swelling, night cramps, and itching compared with those in the control group who underwent sclerotherapy alone. The reduction in these complaints was linked to a greater improvement in quality-of-life indicators in the MPFF group, particularly for pain. The majority of patients in this study were in the 30-50-year age group and $95.6 \%$ were less than 60 years old. The presence of reticular veins and telangiectasias can have a significant impact on patients' quality of life not only in terms of their adverse cosmetic effect but also by the impact that symptoms can have on patients' ability to work and conduct their normal family life. Any treatment that can hasten a patient's recovery and lessen symptom severity is therefore advantageous, particularly in active, working-age individuals.

In this observational study physicians were not restricted to a single technique or sclerosing agent, but performed the procedure according to their usual practice. The rates of hyperpigmentation could therefore be due to a number of factors including treatment technique, sclerosing solution and concentration, patient predisposition, as well as how the authors define hyperpigmentation. Post-sclerotherapy hyperpigmentation was observed in $33.9 \%$ of subjects in the MPFF group and $41.2 \%$ in the control group. Post-injection hyperpigmentation is the most unpleasant and long-lasting complication of sclerotherapy, reflecting the severity of extravasal inflammation. Beneficial effects of MPFF on post-procedure hyperpigmentation have also been observed in previous studies [12-14].

This survey is subject to several limitations. In this observational study subjects were not randomized to treatment. The decision to use MPFF was according to the physicians' usual practice and was generally prescribed to individuals with more severe signs and symptoms. In addition the level of surgical intervention in these patients was greater than in those with less severe disease. To achieve statistically significant differences in this comparative intervention study would not only have required a much larger number of observations but also a standardized technique for the sclerotherapy procedure. In the current study it was stipulated that physicians should use their normal sclerotherapy techniques. Despite this, MPFF treatment was associated with a trend for a reduction in all common sclerotherapy-associated adverse events, which reached statistical significance for hyperpigmentation. The six cases of skin necrosis in the MPFF group were a result of sclerotherapy technical errors and not related to MPFF treatment. A further limitation of this study is that VAS results are only provided for one limb, whereas CVD signs and symptoms are generally bilateral. The quality-of-life indicators, however, are focused on overall improvements related to both limbs.

\section{CONCLUSION}

Phlebosclerosis of dilated intradermal veins results in good aesthetic and functional outcomes and in the majority of patients achieving the lowest CEAP clinical class. MPFF treatment initiated 2 weeks before the procedure and continued for 6 weeks is well accepted by patients and was associated with greater improvements in venous symptoms and quality-of-life indicators compared with the group receiving sclerotherapy alone. In the MPFF group there was also a reduced incidence of typical adverse effects of compression sclerotherapy, especially the occurrence of post-injection hyperpigmentation. On the basis of data from the present study, adjuvant phlebotrophic therapy with MPFF may be considered as an add-on treatment in routine clinical practice when performing phlebosclerosing treatment.

\section{ACKNOWLEDGEMENTS}

We thank all participants of this study for the careful collection and input of data in accordance with the protocol.

Funding. Sponsorship for this study and funding for the article processing charges, open access fee, and editorial assistance for this article were funded by Servier. All authors had full access to all of the data in this study and take 
complete responsibility for the integrity of the data and accuracy of the data analysis.

Editorial Assistance. The authors acknowledge editorial assistance by Freelance Medical Writer Jenny Grice, Le Prioldy, Bieuzy les Eaux, France. This was funded by Servier.

Authorship. All named authors meet the International Committee of Medical Journal Editors (ICMJE) criteria for authorship for this article, had full access to all of the data in this study, take complete responsibility for the integrity of the data and accuracy of the data analysis, and have given their approval for this version to be published.

Authorship Contributions. All authors participated in and contributed to study design, data interpretation, and manuscript preparation.

Disclosures. Vadim Y. Bogachev has conducted lectures and master classes for Servier. Boris V. Boldin and Pavel Y. Turkin have nothing to disclose.

Compliance with Ethics Guidelines. The protocol and design of the study were approved by the local ethics committee of the Russian National Research Medical University named after N.I. Pirogov (Chairmen Storozhakov G.I.) (Extract from Protocol No. 144 dd. 26.01.2016). In accordance with the Federal Law No. 61-FZ, the study did not require the approval of the Ethics Council of the Ministry of Health of the Russian Federation and permission from the Ministry of Health of Russia, as this study had an observational nature. The study was conducted in accordance with the legislative requirements and ethical principles set forth in the Federal Law "On the circulation of medicines" (No. 61-FZ of April 12, 2010, as amended), National Standard of the Russian Federation GOST R 52379-2005 "Good Clinical Practice", World Medical Association's Declaration of Helsinki (1964, with subsequent amendments), and the guidelines for Good Clinical Practice (ICH GCP).
Data Availability. The datasets generated and/or analyzed during the current study are available from the corresponding author on reasonable request.

Open Access. This article is distributed under the terms of the Creative Commons Attribution-NonCommercial 4.0 International License (http://creativecommons.org/licenses/ by-nc/4.0/), which permits any noncommercial use, distribution, and reproduction in any medium, provided you give appropriate credit to the original author(s) and the source, provide a link to the Creative Commons license, and indicate if changes were made.

\section{REFERENCES}

1. Savelyev VS, Kirienko AI, Bogachev VY. Chronic venous diseases in the Russian Federation. The results of the international research program Vein Consult. Phlebology. 2010;4(3):9-12 (article in Russian).

2. Rabe E, Guex JJ, Puskas A, Scuderi A, Fernandez Quesada F, VCP Coordinators. Epidemiology of chronic venous disorders in geographically diverse populations: results from the Vein Consult program. Int Angiol. 2012;31(2):105-15.

3. Rabe E, Breu FX, Cavezzi A, et al. European guidelines for sclerotherapy in chronic venous disorders. Phlebology. 2014;29(6):338-54.

4. Bogachev VY, Boldin BV, Lobanov VN, Arkadan NR, Ermak MY. Adjuvant phlebotrophic therapy and its effect on the pro-inflammatory response after sclerotherapy. Angiol Vasc Surg. 2016;22(4):90-5 (article in Russian).

5. Bogachev V, Golovanova O, Kuznetsov A, Shekoyan A. Can micronized purified flavonoid fraction (MPFF) improve outcomes of lower extremity varicose vein endovenous treatment? First results from the decision study. Phlebolymphology. 2013;20(4):181-7.

6. Bogachev VY, Golovanova OV, Kuznetsov AN, Shekoyan AO. On the rationale of periprocedural phleboprotection in endovascular treatment of varicose veins of the lower extremities. First results of the DECISION study. Angiol Vasc Surg. 2012;18(2):90-5 (article in Russian). 
7. Launois R, Le Moine JG, Lozano FS, Mansilha A. Construction and international validation of CIVIQ-14 (a short form of CIVIQ-20), a new questionnaire with a stable factorial structure. Qual Life Res. 2012;21:1051-8.

8. Le Moine JG, Fiestas-Navarrete L, Katumba K, Launois R. Psychometric validation of the 14 item chronic venous insufficiency quality of life questionnaire (CIVIQ-14): confirmatory factor analysis. Eur J Vasc Endovasc Surg. 2016;51(2):268-74.

9. Darvall KA, Bate GR, Adam DJ, Bradbury AW. Generic health-related quality of life is significantly worse in varicose vein patients with lower limb symptoms independent of CEAP clinical grade. Eur J Vasc Endovasc Surg. 2012;44(3):341-4.

10. Eklöf B, Rutherford RB, Bergan JJ, et al. Revision of the CEAP classification for chronic venous disorders: consensus statement. J Vasc Surg. 2004;40(6):1248-52.
11. Veverkova L, Kalac J, Jedlicka V, Wechsler J. Analysis of the various procedures used in great saphenous vein surgery in Czech Republic and benefit of Daflon $500 \mathrm{mg}$ to postoperative symptoms. Phlebology. 2006;13:193-9.

12. Bogachev V, Boldin B, Lobanov V. Benefits of micronized purified flavonoid fraction as adjuvant therapy on the inflammatory response after sclerotherapy. Int Angiol. 2018;37:71-78.

13. Pitsch F. Benefit of Daflon $500 \mathrm{mg}$ in combination with sclerotherapy of telangiectasias of the lower limbs: results from the SYNERGY and SATISFY surveys. Phlebolymphology. 2011;19:182-7.

14. de Souza Md, Cyrino FZ, Mayall MR, et al. Beneficial effects of the micronized purified flavonoid fraction (MPFF, Daflon ${ }^{\circledR} 500 \mathrm{mg}$ ) on microvascular damage elicited by sclerotherapy. Phlebology. 2016;31:505-6. 